\title{
TWO CHARACTERIZATIONS OF POWER COMPACT OPERATORS
}

\author{
D. G. TACON
}

\begin{abstract}
It is shown that if $T$ is an operator on a Banach space with the property that for every bounded sequence $\left\{x_{n}\right\}$ there is an integer $k$ such that $\left\{T^{k}\left(x_{n}\right)\right\}$ has a convergent subsequence then $T$ is power compact. The equivalent nonstandard characterization is that for each finite point $p$ in an $\boldsymbol{N}_{1}$-saturated enlargement of the space there is an integer $k$ such that $T^{k} p$ is near-standard. Similar results are shown to hold for countable families of operators and for operators possessing weakly compact powers.
\end{abstract}

Let $\left\{T_{n}: n=1,2,3, \ldots\right\}$ be a countable set of bounded linear transformations mapping from the Banach space $X$ into the Banach space $Y$. The main purpose of this note is to show that if, for every bounded sequence $\left\{x_{n}\right\}$ of $X$ there is a positive integer $k$ such that $\left\{T_{k}\left(x_{n}\right)\right\}$ has a convergent (respectively weakly convergent) subsequence in $Y$, then one of the $T_{k}$ is compact (respectively weakly compact). Thus if $X=Y$ and $T_{k}=T^{k}$ for some fixed operator $T$ we obtain the first result of the abstract; recall that $T$ is power compact (or quasi-compact) if there is a positive integer $k$ such that $T^{k}$ is compact. In a Hilbert space setting these operators correspond to the nilpotent elements of the Calkin algebra and are known then to be compact perturbations of nilpotent operators [3]. They also arise naturally as weakly compact operators on such classical spaces as $C(S)$ and $L_{1}(S, \Sigma, \mu)[1$, Chapter VI] and have been extensively studied because, for example, they share many of the spectral properties of compact operators [1, p. 579]. In fact it was the observation that the proofs of these spectral results also held for operators possessing the above (apparently) more general compactness property that led the author to examine the question whether the two classes coincided. We show that they do by providing what seems to be a natural proof which extends to give analogous results for operators with weakly compact powers. This nonstandard proof is based on an application of the Baire category theorem to the nonstandard hull of $X$ and Robinson's characterization of compactness $[4$, p. 93].

We suppose our spaces are embedded in an appropriately large structure

Received by the editors September 24, 1977 and, in revised form, June 19, 1978.

AMS (MOS) subject classifications (1970). Primary 47B05.

Key words and phrases. Banach space, operator, compact, weakly compact, $\aleph_{1}$-saturated enlargement, nonstandard analysis. 
$\Re$, and we develop the nonstandard theory in an $\aleph_{1}$-saturated enlargement * $\Re$ [2]. The nonstandard hull $\hat{X}$ of $X$ is the quotient space fin ${ }^{*} X / \mu(0)$ where fin ${ }^{*} X$ denotes the set of norm-finite elements of ${ }^{*} X$ and $\mu(0)$, the monad of 0 , is the set of norm-infinitesimal elements of ${ }^{*} X$. The hull becomes a normed linear space when given the norm $\|\hat{p}\|=\operatorname{st}(\|p\|)$. Here $\hat{p}$ denotes the equivalence class determined by $p \in$ fin $^{*} X$ in $\hat{X}$ and st denotes the standard part operator. By a result of Luxemburg [2] $\hat{X}$ is complete under the assumption that $* \mathfrak{R}$ is $\boldsymbol{\kappa}_{1}$-saturated. Finally we say $p \in{ }^{*} X$ is weakly near-standard if it is near-standard [4, p. 93] when $X$ is given the weak topology.

THEOREM 1. Suppose the family $\left\{T_{k}: k=1,2,3, \ldots\right\}$ has the property that for each $p \in$ fin ${ }^{*} X$ there is a positive integer $k$ such that $T_{k}(p)$ is near-standard (respectively weakly near-standard). Then there is a positive integer $k$ such that $T_{k}(p)$ is near-standard (respectively weakly near-standard) for each $p \in$ fin ${ }^{*} X$.

Proof. For $k=1,2,3, \ldots$ we define $X_{k}$ to be $\left\{\hat{p}: T_{k} p\right.$ is near-standard (respectively weakly near-standard) $\}$. These are meaningful definitions and as we shall see later each $X_{k}$ is then a closed subspace of $\hat{X}$. By the initial assumptions $\cup_{k=1}^{\infty} X_{k}=\hat{X}$ whence, by the Baire category theorem, $X_{k}=\hat{X}$ for some integer $k$. This implies $T_{k} p$ is near-standard (respectively weakly near-standard) for each $p \in$ fin $^{*} X$. Thus we have the result once we have checked that the $X_{k}$ are closed. For the first case assume $\hat{p}_{n} \rightarrow \hat{p}$ where $\operatorname{st}\left(T_{k}\left(p_{n}\right)\right)=y_{n}$ for each $n$. Then $\left\{y_{n}\right\}$ is a Cauchy sequence in $Y$ since

$$
\left\|y_{l}-y_{m}\right\|=\operatorname{st}\left\|T_{k}\left(p_{l}-p_{m}\right)\right\| \leqslant\left\|T_{k}\right\|\left\|\hat{p}_{l}-\hat{p}_{m}\right\|,
$$

and therefore is convergent as $Y$ is complete. If $y=\lim y_{n}$ then the inequality

$$
\left\|y-T_{k} p\right\| \leqslant\left\|y-y_{n}\right\|+\left\|y_{n}-T_{k} p_{n}\right\|+\left\|T_{k}\left(p_{n}-p\right)\right\|
$$

forces the equality $y=\operatorname{st}\left(T_{k} p\right)$. In the other case assume $\hat{p}_{n} \rightarrow \hat{p}$ where weak-st $\left(T_{k}\left(p_{n}\right)\right)=y_{n}$ for each $n$. This time $\left\{y_{n}\right\}$ is a Cauchy sequence in $Y$ since $\left\|f\left(y_{l}-y_{m}\right)\right\|=\mathrm{st}\left|f\left(T_{k}\left(p_{l}-p_{m}\right)\right)\right| \leqslant\left\|T_{k}\right\|\left\|\hat{p}_{l}-\hat{p}_{m}\right\|$ whenever $f \in$ $S^{\prime}$, the unit sphere of the dual space $Y^{\prime}$. If we let $y=\lim y_{n}$ then $y=$ weakst $T_{k}(p)$.

Corollary 1. Suppose the family $\left\{T_{k}: k=1,2,3, \ldots\right\}$ has the property that for each $p \in$ fin $^{*} X$ there is a positive integer $k$ such that $T_{k}(p)$ is near-standard (respectively weakly near-standard). Then there is a positive integer $k$ such that $T_{k}$ is compact (respectively weakly compact).

Proof. Both conclusions are consequences of the theorem and the earlier referred to result of Robinson.

THEOREM 2. Suppose the family $\left\{T_{k}: k=1,2,3, \ldots\right\}$ has the property that for each bounded sequence $\left\{x_{n}\right\}$ of $X$ there is a positive integer $k$ such that 
$\left\{T_{k}\left(x_{n}\right)\right\}$ has a convergent subsequence. Then for each $p \in$ fin $* X$ there is a positive integer $k$ such that $T_{k} p$ is near-standard.

Proof. Since $Y$ is complete the result holds if we show there is an integer $k$ such that for each $\varepsilon>0$ there exists a point $y \in Y$ such that

$$
\left\|T_{k} p-y\right\|<\varepsilon \text {. }
$$

Let us suppose, to the contrary, that for each $k=1,2,3, \ldots$ there exists a constant $\varepsilon_{k}>0$ such that for each $y \in Y$

$$
\left\|T_{k} p-y\right\|>\varepsilon_{k} .
$$

As a consequence of this we will show that there exists a bounded sequence $\left\{x_{n}\right\}$ in $X$ such that

$$
\left\|T_{k}\left(x_{i}\right)-T_{k}\left(x_{j}\right)\right\|>\varepsilon_{k} \quad \text { for } k=1,2,3, \ldots,
$$

provided $\max (i, j)>k$ and $i \neq j$.

Since the existence of this sequence clearly contradicts the assumption of the theorem we are finished once we have established the existence of the sequence. Suppose in fact that we have chosen $x_{1}, x_{2}, \ldots, x_{k}$ after having initially chosen $x_{1}$ arbitrarily. If $A$ is a standard bound for $\|p\|$ then the (formal) sentence

$$
\exists x \in X\|x\| \leqslant A \&\left\|T_{i} x_{j}-T_{i} x\right\|>\varepsilon_{i} \text { for } i, j=1, \ldots, k
$$

is true in $* \mathfrak{T}$ ( $p$ satisfies it there) and so it is true in $\mathscr{N}$. This guarantees the existence of a point $x_{k+1}$ in $X$ satisfying

$$
\left\|T_{i} x_{j}-T_{i} x_{k+1}\right\|>\varepsilon_{i} \quad \text { for } i, j=1, \ldots, k
$$

and thus ultimately that of the sequence.

Corollary 2. Suppose the family $\left\{T_{k}: k=1,2,3, \ldots\right\}$ has the property that for each bounded sequence $\left\{x_{n}\right\}$ of $X$ there is a positive integer $k$ such that $\left\{T_{k}\left(x_{n}\right)\right\}$ has a convergent subsequence. Then there is a positive integer $k$ such that $T_{k}$ is compact.

Proof. The result is an immediate consequence of Corollary 1 and Theorem 2.

THEOREM 3. Suppose the family $\left\{T_{k}: k=1,2,3, \ldots\right\}$ has the property that for each bounded sequence $\left\{x_{n}\right\}$ of $X$ there is a positive integer $k$ such that $\left\{T_{k}\left(x_{n}\right)\right\}$ has a weakly convergent subsequence. Then for each $p \in$ fin $^{*} X$ there is a positive integer $k$ such that $T_{k} p$ is weakly near-standard.

Proof. Clearly, since $Y$ is complete, it is sufficient to show there exists an integer $k$ such that for each $\varepsilon>0$ there exists a point $y \in Y$ such that for each $f \in S^{\prime}$,

$$
f\left(T_{k} p\right)-f(y)<\varepsilon .
$$

In fact this is equivalent [5, Lemma 2] to showing there exists an integer $k$ such that for each $\varepsilon>0$ there is a finite subset $C$ of $Y$ such that for each 
$f \in S^{\prime}$ there is a point $y \in C$ satisfying

$$
f\left(y-T_{k} p\right)<\varepsilon .
$$

We prove this last assertion by contradiction. Suppose for $k=1,2,3, \ldots$ there exists $\varepsilon_{k}>0$ such that for each finite subset $C$ of $Y$ there is an $f \in S^{\prime}$ such that for each $y \in C, f\left(y-T_{k} p\right)>\varepsilon_{k}$. We claim that as a consequence of this we can construct a bounded sequence $\left\{x_{n}\right\}$ in $X$ and for each integer $k$ a sequence $\left\{f_{n}^{k}: n=k, k+1, k+2, \ldots\right\}$ of elements from $S^{\prime}$ satisfying

$$
f_{n}^{k}\left(T_{k} x_{i}-T_{k} x_{j}\right)>\varepsilon_{k} \quad \text { for } 1<i \leqslant n<j .
$$

To see this suppose we have chosen $x_{i}$ for $i=1, \ldots, k$ and $f_{i}^{j}$ for $1 \leqslant j \leqslant i$ $<k$. Then for $l=1,2, \ldots, k$ we choose functionals $f_{k}^{l}$ satisfying

$$
f_{k}^{l}\left(T_{l}\left(x_{i}\right)-T_{l}(p)\right)>\varepsilon_{l} \text { for } i=1,2, \ldots, k .
$$

Arguing as in Theorem 2 it follows that there must be a suitably bounded element $x_{k+1}$ such that

$$
f_{k}^{l}\left(T_{l}\left(x_{i}\right)-T_{l}\left(x_{k+1}\right)\right)>\varepsilon_{l} \text { for } i, l=1,2, \ldots, k
$$

and this gives the existence of the sequences. The proof is completed once we verify that for each positive integer $l$ the sequence $\left\{T_{l}\left(x_{n}\right)\right\}$ does not have a weakly convergent subsequence. If we suppose that for a given integer $k$, $\left\{T_{k}\left(x_{n}\right)\right\}$ has a weakly convergent subsequence then by renumbering if necessary we can assume there is an element $y \in Y$ such that

$$
T_{k}\left(x_{j}\right) \rightarrow y \quad \text { weakly as } j \rightarrow \infty \text {. }
$$

Then

$$
f_{n}^{k}\left(T_{k} x_{i}-y\right) \geqslant \varepsilon_{k} \text { for } 1 \leqslant i \leqslant n .
$$

But the Banach-Alaoglu theorem ensures that the sequence $\left\{f_{n}^{k}: n=k, k=\right.$ $1, \ldots\}$ has a weak* accumulation point $f$, and hence, using a diagonal process, there exists a subsequence $\left\{n_{p}\right\}$ such that for each $i=1,2, \ldots$

$$
\lim _{p \rightarrow \infty} f_{n_{p}}^{k}\left(T_{k} x_{i}\right)=f\left(T^{k} x_{i}\right) \text {. }
$$

It then follows that $f\left(T_{k} x_{i}-y\right) \geqslant \varepsilon_{k}$ for $i=1,2,3, \ldots$ contradicting the assumption that $T_{k} x_{i} \rightarrow y$ in the weak topology.

COROllaRY 3. Suppose the family $\left\{T_{k}: k=1,2,3, \ldots\right\}$ has the property that for each bounded sequence $\left\{x_{n}\right\}$ of $X$ there is a positive integer $k$ such that $\left\{T_{k}\left(x_{n}\right)\right\}$ has a weakly convergent subsequence. Then there is a positive integer $k$ such that $T_{k}$ is weakly compact.

Proof. This final result is a consequence of Corollary 1 and Theorem 3.

\section{REFERENCES}

1. N. Dunford and J. T. Schwartz, Linear operators, Part I, Interscience, New York, 1958.

2. W. A. J. Luxemburg, A general theory of monads, Applications of Model Theory to Algebra, Analysis, and Probability (Internat. Sympos., Pasadena, Calif., 1967), Holt, Rinehart and Winston, New York, 1969, pp. 18-86. MR $39 \sharp 6244$. 
3. Catherine Olsen, A structure theorem for polynomial compact operators, Amer. J. Math. 93 (1971), 686-698.

4. A. Robinson, Non-standard analysis, Studies in Logic and the Foundation of Math., North-Holland, Amsterdam, 1966. MR 34 \$5680.

5. D. G. Tacon, Weak compactness in normed linear spaces, J. Austral. Math. Soc. 14 (1972), 9-16. MR 47 \$3954.

University OF Newcastle UPON TYNe, Newcastle UPON TYNe, ENGLAND

Universtry of New South Wales, Kensington, N. S. W. 205 Australu (Current address) 